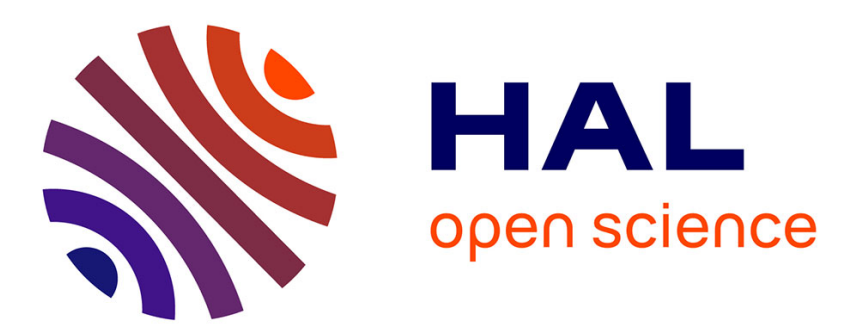

\title{
Increasing Network Lifetime in an Energy-Constrained Wireless Sensor Network
}

Said El Abdellaoui, Mérouane Debbah, Youssef Fakhri, Driss Aboutajdine

\section{To cite this version:}

Said El Abdellaoui, Mérouane Debbah, Youssef Fakhri, Driss Aboutajdine. Increasing Network Lifetime in an Energy-Constrained Wireless Sensor Network. International Journal of Sensor Networks (IJSNET), 2013, 13 (1), pp.44-56. 10.1504/IJSNET.2013.052730 . hal-00769396

\section{HAL Id: hal-00769396 \\ https://hal-centralesupelec.archives-ouvertes.fr/hal-00769396}

Submitted on 1 Jan 2013

HAL is a multi-disciplinary open access archive for the deposit and dissemination of scientific research documents, whether they are published or not. The documents may come from teaching and research institutions in France or abroad, or from public or private research centers.
L'archive ouverte pluridisciplinaire HAL, est destinée au dépôt et à la diffusion de documents scientifiques de niveau recherche, publiés ou non, émanant des établissements d'enseignement et de recherche français ou étrangers, des laboratoires publics ou privés. 
Increasing Network Lifetime in an

Energy-constrained Wireless Sensor Network

\title{
Said El Abdellaoui
}

LRIT unité associée au CNRST (URAC 29),

Faculty of Sciences, Mohammed V-Agdal University,

4 Avenue Ibn Battouta, B.P 1014 RP, Rabat, Morocco

phone: + 212(05) 377718 34/35/38, fax: + 212(05) 37774261 .

E-mail: elabdellaoui.said@yahoo.fr

*Corresponding author

\section{Merouane Debbah}

Alcatel-Lucent Chair on Flexible Radio,

SUPELEC Plateau de Moulon 3, rue Joliot-Curie

Bureau 5-24 91192, Gif-sur-Yvette Cedex, France,

phone: +33 (0) 1698514 47, fax: +33 (0) 169851459 .

E-mail: merouane.debbah@supelec.fr

\section{Youssef Fakhri}

LARIT, équipe Réseaux et Télécommunications, Faculty of Sciences, University Ibn Tofail, B.P 133, Kénitra 14000 ,Morocco phone: + 212 (05) 373294 00, fax: + 212 (05) 37329433.

E-mail: fakhri-Youssef@univ-ibntofail.ac.ma

\section{Driss Aboutajdine}

LRIT unité associée au CNRST (URAC 29), Faculty of Sciences, Mohammed V-Agdal University, 4 Avenue Ibn Battouta B.P 1014 RP, Rabat, Morocco phone: + 212(05) $37771834 / 35 / 38$, fax: + 212(05) 37774261. E-mail: aboutaj@fsr.ac.ma

\begin{abstract}
Energy in Wireless Sensor Networks is a scarce resource, therefore an energyefficient management is required to increase the network lifetime. In this paper, we study the problem of optimal power allocation, taking into account the estimation of total signal-to-noise ratio (SNR) at the Fusion Center (FC). We consider that nodes transmit their data to the Fusion Center over quasi-static Rayleigh fading channels (QSRC). In order to analyze our approach, we will investigate first the orthogonal channels, and secondly the non-orthogonal ones introducing a virtual MISO in the communication. We consider in both cases that the nodes have Channel State Information (CSI). Simulations that have been conducted using these two channel configurations show that, thanks to our new algorithm, the network lifetime is extended by an average that can reach $82,80 \%$ compared to the network lifetime in the other methods.
\end{abstract}

Keywords: MISO Cooperative; Cooperative Communication; QSRC; convex optimization.

\section{Introduction}

Wireless sensor networks (WSNs) represent a technological revolution resulting from convergence of electronic systems and wireless communication systems. A WSN is a special network composed of a large number of nodes equipped with embedded processors, sensors, and radios. These nodes collaborate to accomplish a common task such as environment monitoring or tracking by collecting data and performing local processing. Actually, there are two ways to process sensored data. In the first one, the nodes exchange information (measurements) between them, and based 
on this exchange each one of them gets the estimation of the measured parameter. In the second one, the data are routed over multiple nodes to reach a destination node named the Fusion Center (FC). In our work, we consider the case where all nodes have direct access to the FC.

The WSNs have been widely used in several areas, such as military operations, prevention against fires, public safety. In such areas, the sensors are often randomly placed (they can be dropped from an aircraft). Due to this arbitrary distribution which is frequently done in hostile or inaccessible environments and also due to the small size of sensors, energy becomes a scarce resource in WSNs. This fact automatically influences the lifetime of the sensors and, therefore, the lifetime of the whole network. So, an efficient energy management is necessary to maximize the network lifetime.

In order to solve this problem and put our work in perspective, we give a brief overview of related works. We begin by mentioning the work of (Nguyen et al. 2007) and (Marzetta \& Hochwald 1999) which shows that considering the model of energy presented in (Cui et al. 1999), the total power consumption of a RF system consists of two main sources, namely the transmission power $P_{p a}$ and the power dissipated in the circuit $P_{c}$ by all RF blocks. for simplicity, the blocks of digital signal processing (coding, modulation, detection...) are omitted.

Based on the work in (Chouhan et al. 2009), in longrange applications, the energy efficient management focuses on minimizing the transmission energy only, where the circuit energy is not taken into account. However, in short-range applications the signal transmission energy required is not very high and is comparable to the circuit energy consumed in transmitting and receiving signal. Then, different approaches need to be taken into account to minimize the total energy consumption. To achieve this goal, we mention the work of Collin et al. (Collin et al. 2004) which proves that as to provide a reduced transmission power, the distance between the nodes should be reduced. Consequently, the cooperative approach is used (Nosratinia \& Hedayat 2004)(Hunter \& Nosratinia 2002)(Hunter \& Nosratinia 2003), where information coming from the source node passes by a number of relay nodes before reaching the destination node improving energy efficiency.

Recently, Multi-antenna systems and relay transmission have been fully exploited and intensively studied in wireless networks; this is due to their potential to remarkably decrease the energy consumption. According to Belmega et al in (Belmega et al. 2010), Multi-Input Multi-Output (MIMO) systems are more energy efficient than SISO systems if the only consumed energy that is taken into account is the one used during transmission. However, when the circuitry energy consumption is also considered, this affirmation is no longer true.

Given the limited physical size of sensor nodes, they cannot carry multiple antennas at the same time, and knowing that the energy efficiency of MIMO transmission is particularly useful for WSN, a new transmission technique named "Cooperative MIMO" has been introduced in (Cui et al. 2004) (Jayaweera 2006) (Abdellaoui et al. 2009). This technique is based on the cooperation principle where the existence of different nodes in the network is exploited to transmit the information from the source to a specific destination by virtually using the MIMO system (Shafi et al. 2003)(Sendonaris et al. 2003). The Cooperative MIMO allows to obtain the space-time diversity gain (Winters 1998), the reduction of energy consumption (Nguyen et al. 2007)(Jayaweera 2004), and the enhancement of the system capacity (Gesbert et al. 2003). In our work, we consider a "Cooperative MISO" where all nodes have direct access to the FC.

In (Nguyen et al. 2008), Nguyen et al. propose two techniques of cooperative reception to enhance energy efficiency. The whole space-time combination at the destination node is carried out by the first technique, while the second one independently performs signal processing and space-time combination at each cooperative node.

In (Belmega et al. 2010), Li et al. introduce the low-energy adaptive clustering hierarchy (LEACH) framework to improve the energy-efficiency where the STBC cooperative transmission (Saxena et al. 2010) is applied.

In addition, several studies have explicitly addressed the problem of maximizing the network lifetime using various methods for minimizing the energy consumption. In (Y. Thomas Hou \& Wieselthier 2007) optimal solutions are presented for maximizing a static network lifetime through a graph theoretic approach using static broadcast tree. In (Y. Thomas Hou \& Wieselthier 2007) Thomas et al. have presented an optimal solution for maximizing the network lifetime through a graph theoretic approach using a static multicast tree. In (Chandrakasan 1999) (Shih 2001), the authors have explored the fundamental limits of energy-efficient collaborative data-gathering by deriving upper bounds on the lifetime of increasingly sophisticated sensor networks. Those authors assume that sensor nodes consume energy only when they process, send or receive data. In (Winters 1998), the authors have studied the node density vs. network lifetime trade off for a cellbased energy conservation technique. In (S. Cui \& Bahai 2004), the authors propose an optimal configuration algorithm for the case where the information passes by a single relay to reach the destination creating several boughs. Their objective is to minimize the total transmission power subject to a required error rate at the destination. In this case, the transmission power of the source and all the relays are taken to be fixed and equal. In (Y. Thomas Hou \& Wieselthier 2007), the authors have given an extension for the previous work where they have considered a strategy to enhance the performance of the previous algorithm. They have given an expression for the optimal allocation of transmission power amongst the selected relay nodes, 
whereby the total network power for a given system SER is minimized. In (Winters 1998), the objective of the authors is to devise solutions that maximize network lifetime under such a minimal coverage constraint. Alfieri et al. proposed two solutions in order to maximize the network lifetime: the first one, based on column generation, must run in a centralized way, whereas the second one is based on a heuristic algorithm aiming at a distributed implementation. The main objective of the above mentioned works is to find the transmission powers when the nodes have no knowledge of their future transmissions. Thus, any optimization will have a stochastic nature. In literature, such problem is solved using the dynamic programming (Saxena et al. 2010). The complexity of dynamic programming can be expressed as $\Theta\left(M^{E} Q^{M}\right)$, where $Q$ is the number of quantized levels of channels, $M$ is the number of sensors, and $E$ is the number of possible values for the residual energy of a sensor. Therefore, we are motivated to find a new approach less complex and more efficient in terms of lifetime.

Referring to the mentioned previous works, MIMO system (MISO: simplified version) is important to decrease the transmission power, and knowing that the optimal power allocation depends on the measures of the quality of service (QoS), namely the outage probability, capacity, SNR, and BER. Our work consists then to virtually introduce a MISO system and to consider the SNR constraint by creating a new algorithm less complex and more efficient in terms of lifetime. We use the orthogonal channels and the non-orthogonal one taking into account the total SNR constraint at the FC. In other words, the aim of this approach is to provide the optimal transmission power in order to maximize the network lifetime while guaranteeing the required performance.

This paper is organized as follows. Section II explains our method applied to the orthogonal channels and the non-orthogonal one considering that the nodes have direct access to the FC and the nodes transmit their data over a QSRC where the Channel State Information (CSI) is known by all the nodes. Section III, presents the conducted experiments and the last section concludes the paper.

\section{Optimal power allocation schemes}

In this section, we present our new method in order to maximize the network lifetime under the total SNR constraint at the FC. We consider that the nodes transmit their data over quasi-static Rayleigh fading channels. The following study can be adapted to the context of industrial applications in order to provide more efficient information. We consider that the sensors are distributed in different environments of a big factory where machines are very susceptible to fire. Therefore, these sensors are likely to detect a fire and transmit the information about it to the control center (the Fusion
Center). In the proposed system model, to analyze our new approach, we assume $M$ sensors randomly distributed in the area of interest. When a monitored event occurs, the sensed observation made by an $i^{\text {th }}$ sensor can be written as (Namin \& Nosratinia 2008) :

$$
x_{i}=\theta+n_{i}
$$

, where $\theta$ is the actual parameter being measured and $n_{i}$ is the additive complex Gaussian noise with $n_{i} \sim$ $C \mathcal{N}\left(0, \sigma_{i t}^{2}\right)$. The observation $x_{i}$ is multiplied by the amplification gain $w_{i}$, and then transmitted by the sensors to the FC. Therefore, the transmission power is written as $p_{i}=w_{i}^{2}\left(1+\sigma_{i t}^{2}\right)$ assuming that $E\left[\theta^{2}\right]=1$ where $E[$.$] is the mathematical expectation operator.$

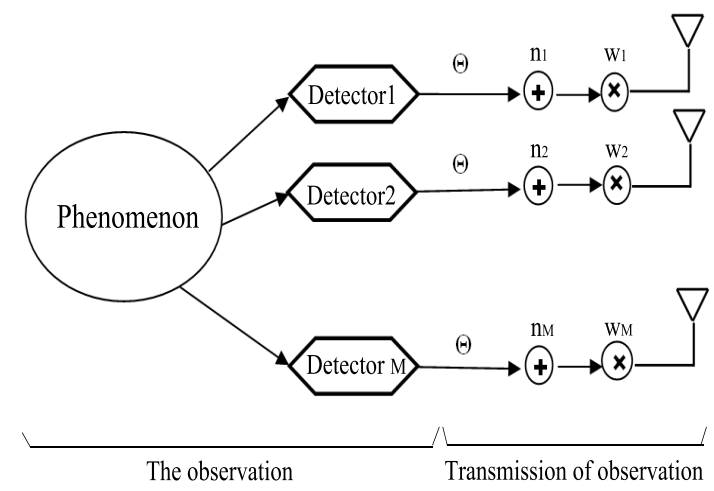

Figure 1 Observation system

\subsection{Orthogonal Channels}

We consider the problem of optimal power allocation for WSNs when using an orthogonal channels between each sensor and the FC taking into account that the nodes have Channel State Information (CSI). In addition, we suppose that a linear minimum mean square-error (LMMSE) detector(Madhow \& Honig 1999) is used at the receiver. This hypothesis will make easier the calculation of SNR at the FC, knowing that the latter is the sum of all the required SNRs from each sensor. In this transmission kind, we determine the SNR corresponding to each sensor in order to meet the overall SNR constraint ensuring that the sum of these SNRs is equal to the total SNR at the FC thanks to the use of the orthogonal channels.

\subsubsection{System Model}

We assume $M$ sensors randomly distributed in the area of interest using an orthogonal channels between the FC and each sensor (see figure 2). The received signal at the FC from $i^{\text {th }}$ sensor is defined by:

$$
y_{i}=h_{i} w_{i}\left(\theta+n_{i}\right)+n_{i r}
$$

where $n_{i r}$ is the noise at the FC with a complex Gaussian distribution $n_{i r} \sim C \mathcal{N}\left(0, \sigma_{i r}^{2}\right)$ and $h_{i}$ is the $i^{t h}$ channel 


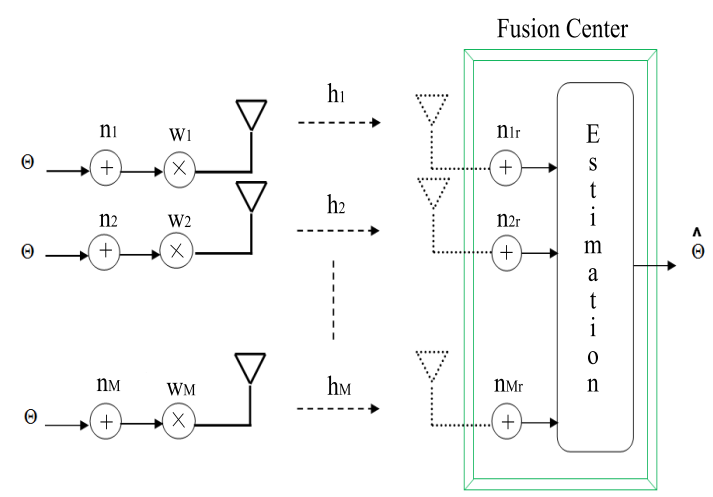

Figure 2 System model using Orthogonal channels

coefficient from the sensor $i$ to the FC. We assume that $\left|h_{i}\right|$ has a Rayleigh distribution.

$$
f\left(\left|h_{i}\right|\right)=\frac{\left|h_{i}\right| e^{\frac{-\left|h_{i}\right|^{2}}{2 \sigma_{h i}^{2}}}}{\sigma_{h i}^{2}}
$$

where $\sigma_{h i}^{2}$ is known.

The SNR corresponding to the $i^{\text {th }}$ sensor using the MMSE detector, as it is detailed in the appendix $\mathrm{D}$, is given by:

$$
S N R_{i}=\frac{p_{i}\left|h_{i}\right|^{2}}{\sigma_{i t}^{2} p_{i}\left|h_{i}\right|^{2}+\left(1+\sigma_{i t}^{2}\right) \sigma_{i r}^{2}}
$$

Since we use the orthogonal channels, the total SNR at the FC will be the sum of all the required SNRs from each sensor. Then, the total SNR of all sensors at the FC can be written as follows:

$$
S N R=\sum_{i=1}^{M} S N R_{i}=\sum_{i=1}^{M}\left[\frac{p_{i}\left|h_{i}\right|^{2}}{\sigma_{i t}^{2} p_{i}\left|h_{i}\right|^{2}+\left(1+\sigma_{i t}^{2}\right) \sigma_{i r}^{2}}\right]
$$

\subsubsection{Problem Formulation}

If channel conditions are measured every $T$ seconds and just before the start of each transmission, and if we consider that the number of transmissions before the network runs out of energy is $N$ (the first node gets depleted), the network lifetime is given by (Namin \& Nosratinia 2008):

$$
\Lambda=N \times T
$$

Hence, to maximize the lifetime of the network it is sufficient to maximize the number of transmissions for each sensor $N$, taking into account the quality constraint defined as the estimation of the SNR at the FC being greater or equal than a target value $\gamma$. Then the formulation of our problem is:

$$
\left\{\begin{array}{c}
\operatorname{Max} N \\
E\left[\sum_{i=1}^{M} S N R_{i j}\right] \geq \gamma, j=1, \ldots, N \\
p_{i j} \geq 0 \\
\forall j
\end{array}\right.
$$

Where $S N R_{i j}$ is the SNR corresponding to the $i^{\text {th }}$ sensor during the $j^{\text {th }}$ transmission period and $p_{i j}$ is the corresponding transmission power during the $j^{\text {th }}$ transmission period. Using the weak law of large numbers (Namin \& Nosratinia 2008) and maintaining that $\sum_{j=1}^{N} p_{i j}<\varepsilon_{i}$, we obtain:

$$
\left\{\begin{array}{c}
\operatorname{Max} N \\
\sum_{j=1}^{N} \sum_{i=1}^{M} S N R_{i j} \geq N \gamma \underset{j}{N}=1, \ldots, N \\
p_{i j} \geq 0 \\
\forall j
\end{array}\right.
$$

where $\varepsilon_{i}$ is an initial energy for the $i^{\text {th }}$ sensor.

The estimated value of the SNR is the average SNR corresponding to the $i^{\text {th }}$ sensor at the FC over network lifetime, given by:

$$
\gamma_{i}=E\left[\frac{p_{i j}\left|h_{i j}\right|^{2}}{\sigma_{i t}^{2} p_{i j}\left|h_{i j}\right|^{2}+\left(1+\sigma_{i t}^{2}\right) \sigma_{i r}^{2}}\right]=E\left[S_{i}\right]
$$

It will be possible to decompose our problem into $M$ single convex optimization problems thanks to the use of the orthogonal channels where the overall SNR at the FC is the sum of all the required SNRs for each sensor. Thus, instead of maximizing the whole network lifetime, we will maximize the individual lifetime of each sensor ensuring that the sum of all the required SNRs of each sensor is equal to the SNR at the FC.

Hence, to maximize the individual lifetime it is adequate to minimize the transmission power. Therefore, the formulation of our problem becomes:

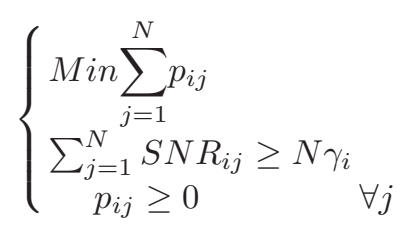

Using the Lagrangian method, we obtain:

$$
\begin{aligned}
& £(p, \lambda, \nu)=\sum_{j=1}^{N} p_{i j}-\sum_{j=1}^{N} \lambda_{j} p_{i j} \\
&+\nu_{i}\left[N \gamma_{i}-\sum_{j=1}^{N} \frac{p_{i j}\left|h_{i j}\right|^{2}}{\sigma_{i t}^{2} p_{i j}\left|h_{i j}\right|^{2}+\left(1+\sigma_{i t}^{2}\right) \sigma_{i r}^{2}}\right]
\end{aligned}
$$

The Karush-Kuhn-Tucker (KKT) conditions (H.Wei \& j. Kubokawa 1998) are as follows:

$$
\left\{\begin{array}{l}
\lambda_{j} \geq 0, \nu_{i} \geq 0, \quad \lambda_{j} p_{i j}=0 \quad \forall j \\
\nu_{i}\left[N \gamma_{i}-\sum_{i=1}^{N}\left[\frac{p_{i j}\left|h_{i j}\right|^{2}}{\sigma_{i t}^{2} p_{i j}\left|h_{i j}\right|^{2}+\left(1+\sigma_{i t}^{2}\right) \sigma_{i r}^{2}}\right]\right]=0 \\
\frac{\partial £}{\partial p_{i j}}=1-\lambda_{j}+\nu_{i}\left[\frac{\sigma_{i r}^{2}\left|h_{i j}\right|^{2}\left(1+\sigma_{i t}^{2}\right)}{\left[\sigma_{i t}^{2} p_{i j}^{2}\left|h_{i j}\right|^{2}+\left(1+\sigma_{i t}^{2}\right) \sigma_{i r}^{2}\right]^{2}}\right]=0
\end{array}\right.
$$

Taking into account this KKT conditions, we conclude that $\nu_{i}>0$ and $\lambda_{j}=0$. Then, we obtain the following solution:

$$
p_{i j}=\frac{\sqrt{\left(1+\sigma_{i t}^{2}\right) \sigma_{i r}^{2}}}{\sigma_{i t}^{2}\left|h_{i j}\right|^{2}}\left[\left|h_{i j}\right| \sqrt{\nu_{i}}-\sqrt{\left(1+\sigma_{i t}^{2}\right) \sigma_{i r}^{2}}\right]
$$

Now, the challenge is to find the value of $\nu_{i}$; for this reason, we must express the $\gamma_{i}=E\left[S_{i}\right]$ in terms of our 
optimal power transmission, and derive the $E\left[p_{i}\right]$. The received $\mathrm{SNR}$ at the $\mathrm{FC}$ from the $i^{\text {th }}$ sensor during the $j^{\text {th }}$ transmission is given by:

$$
S_{i j}= \begin{cases}\frac{1}{\sigma_{i t}^{2}}-\frac{\alpha_{i}}{\sigma_{i t}} \frac{1}{\left|h_{i j}\right|} & \left|h_{i j}\right| \geq \alpha_{i} \\ 0 & \left|h_{i j}\right|<\alpha_{i}\end{cases}
$$

Then, $E\left[S_{i}\right]$ can be expressed by (see Appendix A):

$$
E\left[S_{i}\right]=\frac{1}{\sigma_{i t}^{2}} \exp \left(\frac{-\alpha_{i}^{2}}{2 \sigma_{h i}^{2}}\right)-\frac{\alpha_{i}}{\sigma_{i t}^{2}}\left[\frac{\sqrt{2 \pi}}{2 \sigma_{h i}}\left[1-\operatorname{erf}\left(\frac{\alpha_{i}}{\sqrt{2} \sigma_{h i}}\right)\right]\right]
$$

where $\alpha_{i}=\sqrt{\frac{\left(1+\sigma_{i t}^{2}\right) \sigma_{i r}^{2}}{\nu_{i}}}$ and $\operatorname{erf}()$ is an unilateral error function that is defined as follows:

$$
\operatorname{erf}(x)=\frac{2}{\sqrt{\pi}} \int_{0}^{x} e^{-u^{2}} d u
$$

Then, $p_{i}$ can be expressed in terms of the SNR as follows:

$$
p_{i}=\frac{\alpha_{i} \nu_{i}}{\left|h_{i j}\right|} S_{i j}
$$

Following the same lines as the previous equation, we find:

$E\left[p_{i}\right]=\frac{\sqrt{\pi} \nu_{i} \alpha_{i}}{\sqrt{2} \sigma_{h i} \sigma_{i t}^{2}}\left[1-\operatorname{erf}\left(\frac{\alpha_{i}}{\sqrt{2} \sigma_{h i}}\right)\right]-\frac{\nu_{i} \alpha_{i}^{2}}{2 \sigma_{i t}^{2} \sigma_{h i}^{2}} E_{1}\left[\frac{\alpha_{i}^{2}}{2 \sigma_{h i}^{2}}\right]$

where $E_{n}=\int_{1}^{\infty} \frac{e^{(-x t)}}{t^{n}} d t \quad(x>0, n=0,1,2, \ldots)$ is the exponential integral function.

On the other hand, $\varepsilon_{i}=\sum_{j=1}^{N} p_{i j}=N \times E\left[p_{i}\right]$, assuming that $T=1 s$ then

$$
N=\frac{\varepsilon_{i}}{E\left[p_{i}\right]}
$$

Then, the expected sensor lifetime can be determined as follows:

$$
N=\frac{\varepsilon_{i}}{\frac{\sqrt{\pi} \nu_{i} \alpha_{i}}{\sqrt{2} \sigma_{h i} \sigma_{i t}^{2}}\left[1-\operatorname{erf}\left(\frac{\alpha_{i}}{\sqrt{2} \sigma_{h i}}\right)\right]-\frac{\nu_{i} \alpha_{i}^{2}}{2 \sigma_{i t}^{2} \sigma_{h i}^{2}} E_{1}\left[\frac{\alpha_{i}^{2}}{2 \sigma_{h i}^{2}}\right]}(9)
$$

After finding the expected value of SNR over network lifetime for each sensor, we will assign these last by ensuring that the sum of these SNRs is equal to the total SNR at the FC. In order to satisfy our condition we use the algorithm 1 .

\subsection{Non-Orthogonal Channel}

In this section, we consider that nodes transmit their data to the FC over a QSRC using the Non-Orthogonal channel taking into account that the nodes have Channel State Information (CSI). To illustrate the process, we begin the analysis by considering only two transmitting nodes, afterward we generalize the process by considering $M$ transmitting nodes.

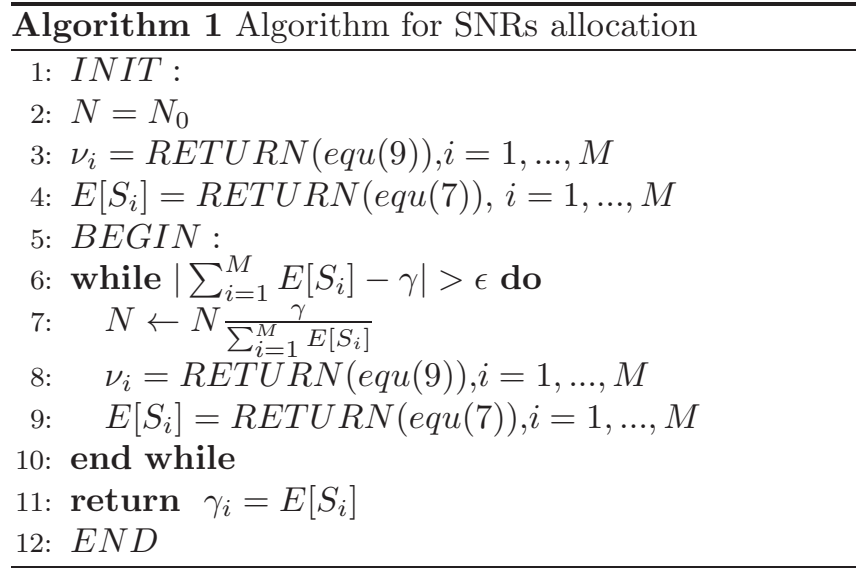

\subsubsection{Virtual MISO $\left(N_{t}=2, N_{r}=1\right)$}

a) System Model

We assume $M$ sensors randomly distributed in the area of interest using Non-Orthogonal channels between the $\mathrm{FC}$ and each sensor and considering that the FC has CSI. We begin the analysis by considering only two transmitting nodes where $N_{t}=2$ and $N_{r}=1$.

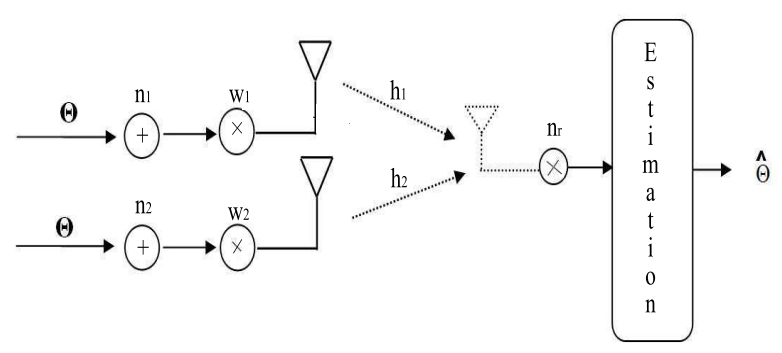

Figure 3 System Model

The received signal at the $\mathrm{FC}$ is defined by:

$$
y=h_{1} w_{1}\left(\theta+n_{1}\right)+h_{2} w_{2}\left(\theta+n_{2}\right)+n_{r}
$$

where $h_{1}$ (respectively $h_{2}$ ) is the channel coefficient from the $1^{\text {st }}$ sensor (respectively $2^{\text {nd }}$ sensor) to the FC (see figure 3) such as these coefficients are Independently Identically Distributed (i.i.d). We assume that $\left|h_{i}\right|$ has a Rayleigh distribution:

$$
f\left(\left|h_{i}\right|\right)=\frac{\left|h_{i}\right| e^{\frac{-\left|h_{i}\right|^{2}}{2 \sigma_{h i}^{2}}}}{\sigma_{h i}^{2}}
$$

where $\sigma_{h i}^{2}$ is known.

Assuming that we use real channels and following the same lines as in Appendix D, the SNR at the FC corresponding to both sensors using the MMSE detector is given by:

$$
S N R=\frac{\left(\left|h_{1}\right| w_{1}+\left|h_{2}\right| w_{2}\right)^{2}}{\sigma_{1 t}^{2} w_{1}^{2}\left|h_{1}\right|^{2}+\sigma_{r}^{2}+\sigma_{2 t}^{2} w_{2}^{2}\left|h_{2}\right|^{2}}
$$


b) Problem Formulation

Maximizing the network lifetime relies on minimizing the amplifications gain at the $l^{t h}$ instant; $w_{1}^{(l)}$ and $w_{2}^{(l)}$.

In this case, our problem can be formulated as:

$$
\left\{\begin{array}{c}
\operatorname{Min} w_{1}^{(l)^{2}}\left(1+\sigma_{1 t}^{2}\right)+w_{2}^{(l)^{2}}\left(1+\sigma_{2 t}^{2}\right) \\
S N R^{(l)} \geq \gamma \quad l=1, \ldots, N \\
w_{i}^{(l)} \geq 0 \quad \forall l
\end{array}\right.
$$

Consequently, the Lagrangian $£$ can be written as follows:

$$
\begin{array}{r}
£\left(w^{(l)}, \lambda, \nu\right)=w_{1}^{(l)^{2}}\left(1+\sigma_{1 t}^{2}\right)+w_{2}^{(l)^{2}}\left(1+\sigma_{2 t}^{2}\right)-\lambda_{1} w_{1}^{(l)} \\
-\lambda_{2} w_{2}^{(l)}+\nu_{l}\left[\gamma\left(w_{1}^{(l)^{2}}\left|h_{1}^{(l)}\right|^{2} \sigma_{1 t}^{2}+\sigma_{r}^{2}+w_{2}^{(l)^{2}}\left|h_{2}^{(l)}\right|^{2} \sigma_{2 t}^{2}\right)\right] \\
-\nu_{l}\left[\left(w_{1}^{(l)}\left|h_{1}^{(l)}\right|+w_{2}^{(l)}\left|h_{2}^{(l)}\right|\right)^{2}\right]
\end{array}
$$

The Karush-Kuhn-Tucker (KKT) conditions (H.Wei \& j. Kubokawa 1998) are as follows:

$$
\left\{\begin{array}{l}
\lambda_{i} \geq 0, \nu_{l} \geq 0, \quad \lambda_{i} w_{i}^{(l)}=0 \quad \forall l, i=1,2 \\
\nu_{l}\left[\gamma\left(w_{1}^{(l)}\left|h_{1}^{(l)}\right|^{2} \sigma_{1 t}^{2}+\sigma_{r}^{2}+\left.\left.w_{2}^{(l)}\right|^{2} h_{2}^{(l)}\right|^{2} \sigma_{2 t}^{2}\right)\right. \\
\left.-\left(w_{1}^{(l)}\left|h_{1}^{(l)}\right|+w_{2}^{(l)}\left|h_{2}^{(l)}\right|\right)^{2}\right]=0 \\
\frac{\partial £}{\partial w_{i}^{(l)}}=2 w_{i}^{(l)}\left(1+\sigma_{i t}^{2}\right)-\lambda_{i}+2 \nu_{l} \gamma\left|h_{i}^{(l)}\right|^{2} w_{i}^{(l)} \sigma_{i t}^{2} \\
-2 \nu_{l}\left|h_{i}^{(l)}\right|\left[\left(w_{i}^{(l)}\left|h_{i}^{(l)}\right|+w_{k \neq i}^{(l)}\left|h_{k \neq i}^{(l)}\right|\right)\right]=0
\end{array}\right.
$$

Taking into account the KKT conditions, we find that $\nu_{l}>0$ and $\lambda_{1}=\lambda_{2}=0$. Then, we obtain the following solution:

$$
\left\{\begin{array}{l}
w_{1}^{(l)}=\sqrt{\frac{\gamma \sigma_{r}^{2} \Upsilon_{2}^{2}}{\left|h_{1}^{(l)}\right|^{2}\left[\left(1+\sigma_{1 t}^{2}\right) \Upsilon_{2}^{2}+\left(\Upsilon_{2}-1+\sigma_{i r}^{2}\right)+2 \nu_{l} \Upsilon_{2}\left|h_{2}^{(l)}\right|^{2}\right]}} \\
w_{2}^{(l)}=\sqrt{\frac{\gamma \sigma_{r}^{2} \Upsilon_{1}^{2}}{\left|h_{2}^{(l)}\right|^{2}\left[\left(1+\sigma_{2 t}^{2}\right) \Upsilon_{1}^{2}+\left(\Upsilon_{1}-1+\sigma_{r}^{2}\right)+2 \nu_{l} \Upsilon_{1}\left|h_{1}^{(l)}\right|^{2}\right]}}
\end{array}\right.
$$

We denote that $\Upsilon_{2}=\left[\nu_{l}\left|h_{2}^{(l)}\right|^{2}\left(\sigma_{2 t}^{2} \gamma-1\right)+\left(1+\sigma_{2 t}^{2}\right)\right]$ and $\Upsilon_{1}=\left[\nu_{l}\left|h_{1}^{(l)}\right|^{2}\left(\sigma_{1 t}^{2} \gamma-1\right)+\left(1+\sigma_{1 t}^{2}\right)\right]$.

The Lagrangian parameter $\nu_{l}$, as it is detailed in the appendix B, can be written as follows:

$$
\begin{aligned}
\nu_{l}=\frac{\sqrt{\left[\nu_{l}\left|h_{1}^{(l)}\right|^{2}\left(\sigma_{1 t}^{2} \gamma-1\right)+\left(1+\sigma_{1 t}^{2}\right)\right]}}{\left|h_{1}^{(l)}\right|} \\
* \frac{\sqrt{\left[\nu_{l}\left|h_{1}^{(l)}\right|^{2}\left(\sigma_{2 t}^{2} \gamma-1\right)+\left(1+\sigma_{2 t}^{2}\right)\right]}}{\left|h_{2}^{(l)}\right|}
\end{aligned}
$$

Note that $\nu_{l}$ can be found numerically using the "Fminsearch" (J.C.Lagarias et al. 1998) function in MATLAB.

\subsubsection{Virtual MISO (Generalized: $N_{t}=M$ )}

In this section, we consider the same assumptions as in the previous section, and generalize the process by considering $M$ transmitting nodes taking into account that the nodes have Channel State Information (CSI) (see figure4). In this case, the channel condition is known by the transmitters, this requires some energy to be spent on energy acquisition.

a) System Model

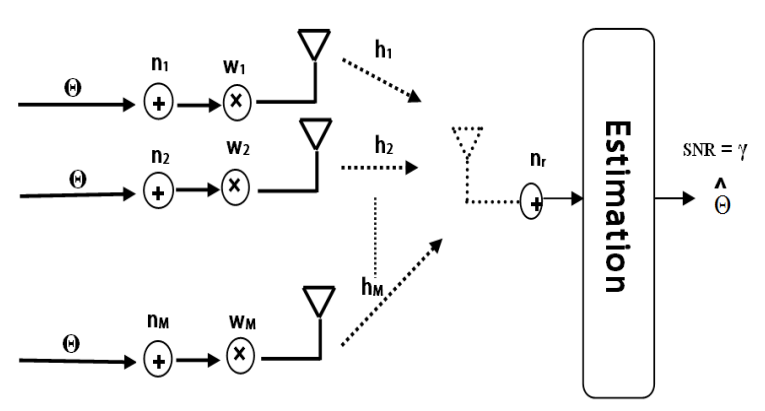

Figure 4 System Model

The received signal at the $\mathrm{FC}$ from $i^{\text {th }}$ sensor is defined by:

$$
y=\sum_{i=1}^{M} h_{i} w_{i}\left(\theta+n_{i}\right)+n_{r}
$$

where $h_{i}$ is the $i^{\text {th }}$ channel coefficient from the sensor $i$ to the fusion center such that these coefficients are i.i.d distributed. We assume that $\left|h_{i}\right|$ has a Rayleigh distribution where $\sigma_{h i}^{2}$ represents the well known variance. Where

$$
f\left(\left|h_{i}\right|\right)=\frac{\left|h_{i}\right| e^{\frac{-\left|h_{i}\right|^{2}}{2 \sigma_{h i}^{2}}}}{\sigma_{h i}^{2}}
$$

Assuming that we use real channels and following the same lines as in Appendix D, the SNR at the FC corresponding to $M$ sensors using the MMSE detector is given by:

$$
S N R=\frac{\left(\sum_{i=1}^{M}\left|h_{i}\right| w_{i}\right)^{2}}{\sum_{i=1}^{M} w_{i}^{2}\left|h_{i}\right|^{2} \sigma_{i t}^{2}+\sigma_{r}^{2}}
$$

Our aim is to maximize the batteries lifetime duration while keeping the expected value of SNR greater than or equal to a target value $\gamma$.

b) Problem Formulation

At the $l^{\text {th }}$ instant, maximizing the lifetime relies on minimizing the power consumption, therefore the problem formulation is given as follows:

$$
\left\{\begin{array}{c}
\operatorname{Min} \sum_{i=1}^{M} w_{i}^{(l)^{2}}\left(1+\sigma_{i t}^{2}\right) \\
S N R^{(l)} \geq \gamma \quad l=1, \ldots, N ; \\
P_{i} \geq 0
\end{array}\right.
$$


To find the optimal points, we use the Lagrange method while satisfying the constraints quoted before. The Lagrangian $£$ can be written as follows:

$$
\begin{gathered}
£\left(w_{i}^{(l)}, \lambda, \nu\right)=\sum_{i=1}^{M} w_{i}^{(l)^{2}}\left(1+\sigma_{i t}^{2}\right)-\sum_{i=1}^{M} \lambda_{i} w_{i}^{(l)} \\
+\nu_{l}\left[\gamma\left[\sum_{i=1}^{M} w_{i}^{(l)^{2}}\left|h_{i}^{(l)}\right|^{2} \sigma_{i t}^{2}+\sigma_{r}^{2}\right]-\left[\sum_{i=1}^{M} w_{i}^{(l)}\left|h_{i}^{(l)}\right|\right]^{2}\right]
\end{gathered}
$$

Let us consider the Karush-Kuhn-Tucker (KKT) conditions for the problem:

$$
\left\{\begin{array}{l}
\lambda_{i} \geq 0, \quad \nu_{l} \geq 0, \lambda_{i} w_{i}^{(l)}=0 \\
\nu_{l}\left[\gamma\left[\sum_{i=1}^{M} w_{i}^{(l)}{ }^{2}\left|h_{i}^{(l)}\right|^{2} \sigma_{i t}^{2}+\sigma_{r}^{2}\right]-\left[\sum_{i=1}^{M} w_{i}^{(l)}\left|h_{i}^{(l)}\right|\right]^{2}\right]=0 \\
\quad \frac{\partial £}{\partial w_{k}^{(l)}}=0
\end{array}\right.
$$

Then, the partial derivative of $£$ with respect to $w_{k}$ is:

$$
\begin{aligned}
\frac{\partial £}{\partial w_{k}^{(l)}}=2 w_{k}^{(l)}\left(1+\sigma_{k t}^{2}\right) & -\lambda_{k}+2 \nu_{l} \gamma\left|h_{k}^{(l)}\right|^{2} \sigma_{k t}^{2} w_{k}^{(l)} \\
& -2 \nu_{l}\left|h_{k}^{(l)}\right|\left(\sum_{i=1}^{M} w_{i}^{(l)}\left|h_{i}^{(l)}\right|\right)
\end{aligned}
$$

Taking into account the KKT conditions, we find that $\nu_{l}>0$ and $\lambda_{k}=0$. Thus,

$$
w_{k}^{(l)}=\frac{\nu_{l}\left|h_{k}^{(l)}\right|\left(\sum_{i=1}^{M} w_{i}^{(l)}\left|h_{i}^{(l)}\right|\right)}{\left(1+\sigma_{k t}^{2}\right)+\nu_{l} \gamma\left|h_{k}^{(l)}\right|^{2} \sigma_{k t}^{2}}
$$

We refer to appendix $\mathrm{C}$ for the details, Equation (20) becomes:

$$
\begin{aligned}
w_{k}^{(l)} & =\frac{\nu_{l}\left|h_{k}^{(l)}\right|}{\left[\left(1+\sigma_{k t}^{2}\right)+\nu_{l} \gamma\left|h_{k}^{(l)}\right|^{2} \sigma_{k t}^{2}\right]} \\
& * \frac{\sigma_{r} \sqrt{\gamma}}{\sqrt{1-\gamma \nu_{l}^{2}\left[\sum_{i=1}^{M} \frac{\left|h_{i}^{(l)}\right|^{4}}{\left[\left(1+\sigma_{i t}^{2}\right)+\nu_{l} \gamma\left|h_{i}^{(l)}\right|^{2} \sigma_{i t}^{2}\right]^{2}} \sigma_{i t}^{2}\right]}}
\end{aligned}
$$

where

$$
\left[\sum_{i=1}^{M} w_{i}^{(l)}\left|h_{i}^{(l)}\right|\right]^{2}=\frac{\gamma \sigma_{r}^{2}}{1-\gamma \nu_{l}^{2}\left[\sum_{i=1}^{M} \frac{\left|h_{i}^{(l)}\right|^{4}}{\left[\left(1+\sigma_{i t}^{2}\right)+\nu_{l} \gamma\left|h_{i}^{(l)}\right|^{2} \sigma_{i t}^{2}\right]^{2}} \sigma_{i t}^{2}\right]}
$$

The Lagrangian parameter $\nu_{l}$, as it is detailed in the appendix B, can be written as follows:

$$
\sum_{i=1}^{M} \frac{\left|h_{k}^{(l)}\right|^{2}}{1+\sigma_{k t}^{2}\left(1+\nu_{l} \gamma\left|h_{k}^{(l)}\right|^{2}\right)}=\frac{1}{\nu_{l}}
$$

This equation is not written in a closed-form solution. Therefore, it can be solved numerically using the function "fminsearch" (J.C.Lagarias et al. 1998).

\section{Simulation and Discussion}

Several simulations have been conducted using MATLAB in order to compare and evaluate the behavior of our novel approach. In the order to demonstrate the performance of our new algorithm, we will compare it to three methods, namely the EP method (Equal Power) in which we attribute the power to each sensor according to its residual energy (Goudarzi \& Pakravan 2008), the MTTP method (Minimum Total Transmission Power) which consists of minimizing the total transmission power across all the nodes while satisfying a distortion requirement on the joint estimate $(\mathrm{S}$. Cui \& Poor 2005), and the U.F method (Utility Function) which is defined as a measure of the mutual satisfaction of maximizing throughput (number of information bits delivered accurately) and minimizing energy consumption (Belmega et al. 2010) (Abdellaoui et al. 2009). For each simulation, we study the network lifetime while increasing the number of nodes. The simulations parameters are generated randomly such that each parameter $\mathrm{p}$ belongs to a uniform distribution between $\psi$ and $\varphi, p \in U[\psi, \varphi]$. These parameters are summarized in tables representing the simulation parameters.

\subsection{Evaluation of the Proposed Method in the Orthogonal channels case.}

Figure 5 shows the behavior of the lifetime network while increasing the number of nodes using orthogonal channels. As can be seen, the proposed approach outperforms other methods concerning the network lifetime. Actually, the network lifetime is extended by an average that can reach $70,52 \%$ thanks to the use of the orthogonal channels where the overhead SNR at the FC is the sum of the SNRs coming from each sensor. The figure reports the robustness of the proposed method since it achieves always better results for all number of sensors. In fact, the proposed method offers an improvement between 1 and 10 sensors that can attempt $20 \%$. Beyond 15 sensors the improvement becomes more important and reaches the maximum value at 70 sensors. Table 1 shows the parameters used for simulations.

\begin{tabular}{|c|l|}
\hline Estimate & Parameters \\
\hline$U[0.1,0.4]$ & $\sigma_{h i}^{2}:$ The variances of channel estimation \\
\hline$U[0.2,0.4]$ & $\sigma_{i r}^{2}:$ The noise variances at the FC \\
\hline$U[0.02,0.2]$ & $\sigma_{i t}^{2}:$ The observation noise variances \\
\hline$U[200,500]$ & $\varepsilon_{i}:$ The initial energy \\
\hline
\end{tabular}

Table 1 Simulations parameters

\subsection{Evaluation of the Proposed Method in Non-Orthogonal channels}

\subsubsection{Virtual MISO Configuration $\left(N_{t}=2\right)$}

Our new method is more effective than the EP method concerning network lifetime. The batteries lifetime 


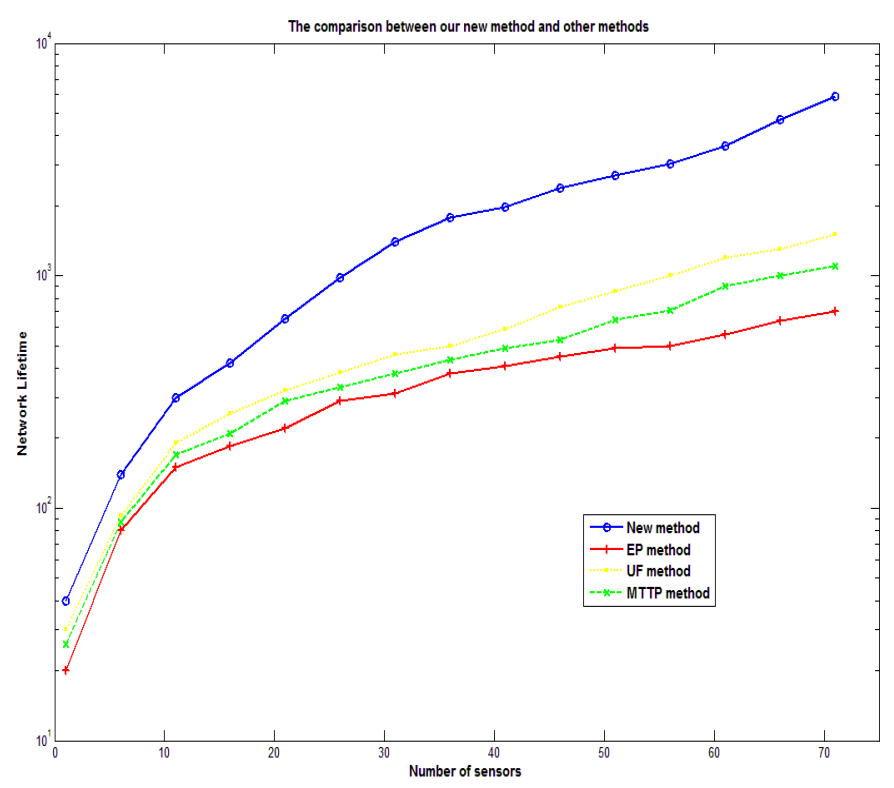

Figure 5 The comparison between our method and other methods concerning network lifetime using the orthogonal channels

duration is extended by an average of $76,30 \%$. Table 2 shows the parameters used for simulations.

\begin{tabular}{|c|l|}
\hline Estimate & Parameters \\
\hline$U[0.1,0.2]$ & $\sigma_{h i}^{2}:$ The variances of channel estimation \\
\hline 0.05 & $\sigma_{r}^{2}:$ The noise variance at the FC \\
\hline$U[0.02,0.1]$ & $\sigma_{i t}^{2}:$ The observation noise variances \\
\hline$U[200,500]$ & $\varepsilon_{i}:$ The initial energy \\
\hline
\end{tabular}

Table 2 Simulations parameters

\subsubsection{Virtual MISO Configuration (Generalized)}

Figure 6 depicts the behavior of the network lifetime while increasing the number of nodes. It is observed that using the proposed method increases the network lifetime by an average that can reach $82,80 \%$ compared to the one obtained by other methods. Actually, the curves show that the network lifetime is clearly extended when the number of sensors exceeds 8 . While between 1 and 8 sensors, the improvement is less important in terms of network lifetime. Table 3 shows the parameters used for simulations.

\begin{tabular}{|c|l|}
\hline Estimate & Parameters \\
\hline$U[0.1,0.2]$ & $\sigma_{h i}^{2}:$ The variances of channel estimation \\
\hline 0.05 & $\sigma_{r}^{2}:$ The noise variance at the FC \\
\hline$U[0.02,0.1]$ & $\sigma_{i t}^{2}:$ The observation noise variances \\
\hline$U[200,500]$ & $\varepsilon_{i}:$ The initial energy \\
\hline
\end{tabular}

Table 3 Simulations parameters

\section{Conclusion}

This paper presents a new algorithm which aims at minimizing the power consumption and consequently

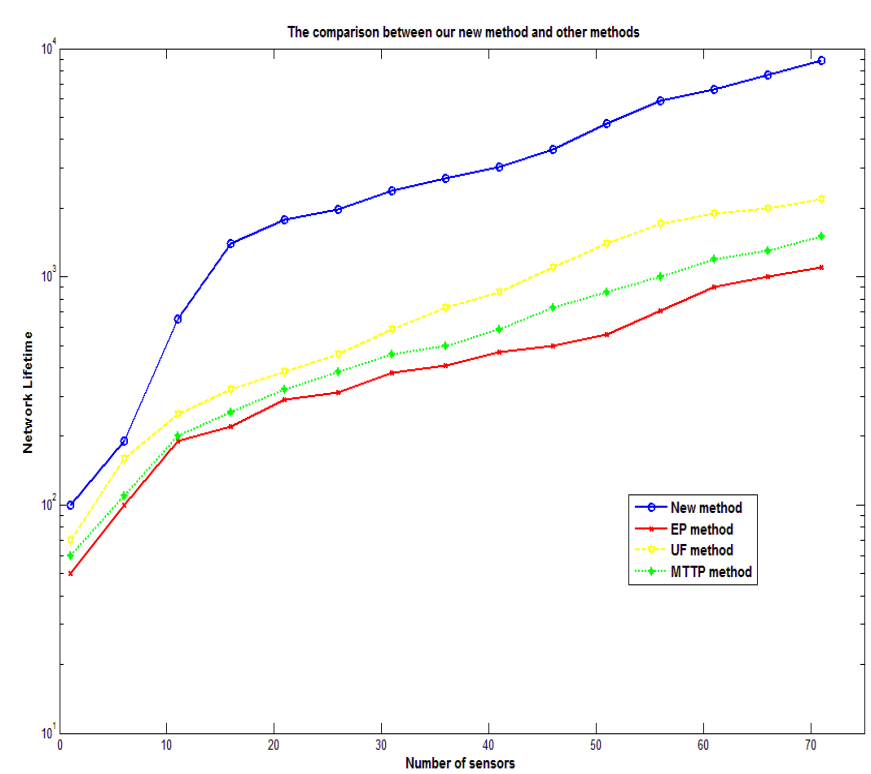

Figure 6 The comparison between our method and other methods concerning the network lifetime using the Non-Orthogonal channels

maximize the network lifetime. This method takes into consideration the estimation of the overall SNR at the FC.

Our simulations show that our new method using the orthogonal channels scheme and Non-Orthogonal one, consumes less energy than the other methods. The future work is to apply our new method to Non-Orthogonal channels assuming that the channel coefficients are unknown.

\section{Appendix A}

In order to find the value of $\nu_{i}$, we must express the $\gamma_{i}=E\left[S_{i}\right]$ in terms of the optimal power transmission scheme, given by equation (7), and then derive the $E\left[p_{i}\right]$. The received SNR at the FC from the $i^{t h}$ sensor during the $j^{\text {th }}$ transmission is given by:

$$
S_{i j}= \begin{cases}\frac{1}{\sigma_{i t}^{2}}-\frac{\alpha_{i}}{\sigma_{i t}^{2}} \frac{1}{\left|h_{i j}\right|} & \left|h_{i j}\right| \geq \alpha_{i} \\ 0 & \left|h_{i j}\right|<\alpha_{i}\end{cases}
$$

where $\alpha_{i}=\sqrt{\frac{\left(1+\sigma_{i t}^{2}\right) \sigma_{i r}^{2}}{\nu_{i}}}$

For calculate the $E\left[S_{i}\right]$, we consider the following lemma (Namin \& Nosratinia 2008) knowing that the random variable $\mathrm{Y}$ is defined in terms of another random variable $\mathrm{X}$ :

then,

$$
\varphi=\left\{\begin{array}{cr}
C+g(X) & X>b \\
0 & X<b
\end{array}\right.
$$

$$
E[\varphi]=C \int_{b}^{\infty} f(x) d x+\int_{b}^{\infty} f(x) g(x) d x
$$

where $f(x)$ is the pdf of $X$.

Then, $E\left[S_{i}\right]$ can be expressed by:

$$
E\left[S_{i}\right]=\frac{1}{\sigma_{i t}^{2}} \exp \left(\frac{-\alpha_{i}^{2}}{2 \sigma_{h i}^{2}}\right)-\frac{\alpha_{i}}{\sigma_{i t}^{2}} \int_{\alpha_{i}}^{\infty} \frac{1}{\sigma_{h i}^{2}} \exp \left(\frac{-x^{2}}{2 \sigma_{h i}^{2}}\right) d x
$$


Then,

$E\left[S_{i}\right]=\frac{1}{\sigma_{i t}^{2}} \exp \left(\frac{-\alpha_{i}^{2}}{2 \sigma_{h i}^{2}}\right)-\frac{\alpha_{i}}{\sigma_{i t}^{2}}\left[\frac{\sqrt{2 \pi}}{2 \sigma_{h i}}\left[1-\operatorname{erf}\left(\frac{\alpha_{i}}{\sqrt{2} \sigma_{h i}}\right)\right]\right.$

where $\operatorname{erf}()$ is an unilateral error function that is defined as follows:

$$
\operatorname{erf}(x)=\frac{2}{\sqrt{\pi}} \int_{0}^{x} \exp -u^{2} d u
$$

Hence,

$E\left[p_{i}\right]=\frac{\sqrt{\pi} \nu_{i} \alpha_{i}}{\sqrt{2} \sigma_{h i} \sigma_{i t}^{2}}\left[1-\operatorname{erf}\left(\frac{\alpha_{i}}{\sqrt{2} \sigma_{h i}}\right)\right]-\frac{\nu_{i} \alpha_{i}^{2}}{2 \sigma_{i t}^{2} \sigma_{h i}^{2}} E_{1}\left[\frac{\alpha_{i}^{2}}{2 \sigma_{h i}^{2}}\right]$

where $E_{n}=\int_{1}^{\infty} \frac{\exp (-x t)}{t^{n}} d t(x>0, n=0,1,2, \ldots)$ is the exponential integral function. From (5), the expected sensor lifetime can be determined as follows:

$$
N=\frac{\varepsilon_{i}}{\frac{\sqrt{\pi} \nu_{i} \alpha_{i}}{\sqrt{2} \sigma_{h i} \sigma_{i t}^{2}}\left[1-\operatorname{erf}\left(\frac{\alpha_{i}}{\sqrt{2} \sigma_{h i}}\right)\right]-\frac{\nu_{i} \alpha_{i}^{2}}{2 \sigma_{i t}^{2} \sigma_{h i}^{2}} E_{1}\left[\frac{\alpha_{i}^{2}}{2 \sigma_{h i}^{2}}\right]}
$$

\section{Appendix B}

Taking into account the KKT conditions, we find that $\nu_{l}>0$ and $\lambda_{1}=\lambda_{2}=0$. The partial derivative of $£$ with respect to $w_{1}$ and $w_{2}$ :

$$
\begin{aligned}
\frac{\partial £}{\partial w_{1}^{(l)}}= & 2 w_{1}^{(l)}\left(1+\sigma_{1 t}^{2}\right)-\lambda_{1}+2 \nu_{l} \gamma\left|h_{1}^{(l)}\right|^{2} w_{1}^{(l)} \sigma_{1 t}^{2} \\
& -2 \nu_{l}\left|h_{1}^{(l)}\right|\left[\left(w_{1}^{(l)}\left|h_{1}^{(l)}\right|+w_{2}^{(l)}\left|h_{2}^{(l)}\right|\right)\right]=0 \\
\frac{\partial £}{\partial w_{2}^{(l)}}= & 2 w_{2}^{(l)}\left(1+\sigma_{2 t}^{2}\right)-\lambda_{2}+2 \nu_{l} \gamma\left|h_{2}^{(l)}\right|^{2} w_{2}^{(l)} \sigma_{2 t}^{2} \\
& -2 \nu_{l}\left|h_{2}^{(l)}\right|\left[\left(w_{2}^{(l)}\left|h_{2}^{(l)}\right|+w_{1}^{(l)}\left|h_{1}^{(l)}\right|\right)\right]=0
\end{aligned}
$$

Thus,

$$
\begin{aligned}
& w_{1}^{(l)}=\frac{\nu_{l}\left|h_{2}^{(l)}\right| w_{2}^{(l)}\left|h_{1}^{(l)}\right|}{\nu_{l}\left|h_{1}^{(l)}\right|^{2}\left(\sigma_{1 t}^{2} \gamma-1\right)+\left(1+\sigma_{1 t}^{2}\right)} \\
& w_{2}^{(l)}=\frac{\nu_{l}\left|h_{1}^{(l)}\right| w_{1}^{(l)}\left|h_{2}^{(l)}\right|}{\nu_{l}\left|h_{2}^{(l)}\right|^{2}\left(\sigma_{2 t}^{2} \gamma-1\right)+\left(1+\sigma_{2 t}^{2}\right)}
\end{aligned}
$$

To calculate $\nu_{l}$, we replace (27) in (28) and get:

$$
\begin{aligned}
w_{1}^{(l)}=\frac{\nu_{l}\left|h_{2}^{(l)}\right|\left|h_{1}^{(l)}\right|}{\nu_{l}\left|h_{1}^{(l)}\right|^{2}\left(\sigma_{1 t}^{2} \gamma-1\right)+\left(1+\sigma_{1 t}^{2}\right)} & \\
& \quad \frac{\nu_{l}\left|h_{1}^{(l)}\right| w_{1}^{(l)}\left|h_{2}^{(l)}\right|}{\nu_{l}\left|h_{2}^{(l)}\right|^{2}\left(\sigma_{2 t}^{2} \gamma-1\right)+\left(1+\sigma_{2 t}^{2}\right)}
\end{aligned}
$$

Then, we divide by $w_{1}$ and obtain:

$$
\begin{aligned}
\nu_{l}=\frac{\sqrt{\left[\nu_{l}\left|h_{1}^{(l)}\right|^{2}\left(\sigma_{1 t}^{2} \gamma-1\right)+\left(1+\sigma_{1 t}^{2}\right)\right]}}{\left|h_{1}^{(l)}\right|} \\
* \frac{\sqrt{\left[\nu_{l}\left|h_{1}^{(l)}\right|^{2}\left(\sigma_{2 t}^{2} \gamma-1\right)+\left(1+\sigma_{2 t}^{2}\right)\right]}}{\left|h_{2}^{(l)}\right|}
\end{aligned}
$$

Note that $\nu_{l}$ can be found numerically using the "Fminsearch" (J.C.Lagarias et al. 1998) function in Matlab. Now, we must search $w_{1}=f\left(\nu_{l}\right)$ and $w_{2}=$ $f\left(\nu_{l}\right)$.

From (11), we have:

$\gamma\left[\sigma_{1 t}^{2} w_{1}^{(l)^{2}}\left|h_{1}^{(l)}\right|^{2}+\sigma_{i r}^{2}+\sigma_{2 t}^{2} w_{2}^{(l)^{2}}\left|h_{2}^{(l)}\right|^{2}\right]=\left(\left|h_{1}^{(l)}\right| w_{1}+\left|h_{2}^{(l)}\right| w_{2}^{(l)^{2}}\right.$

Hence,

$$
\begin{aligned}
\gamma \sigma_{i r}^{2}=w_{1}^{(l)^{2}}\left|h_{1}^{(l)}\right|^{2}\left[1-\gamma \sigma_{1 t}^{2}\right] & +w_{2}^{(l)^{2}}\left|h_{2}^{(l)}\right|^{2}\left[1-\gamma \sigma_{2 t}^{2}\right] \\
& +2 w_{1}^{(l)} w_{2}^{(l)}\left|h_{1}^{(l)}\right|\left|h_{2}^{(l)}\right|
\end{aligned}
$$

We replace (27) in (31), and find:

$$
\begin{gathered}
\frac{\nu_{l}^{2}\left|h_{2}^{(l)}\right|^{4}\left|h_{1}^{(l)}\right|^{2} w_{1}^{(l)^{2}}}{\left[\nu_{l}\left|h_{2}^{(l)}\right|^{2}\left(\sigma_{2 t}^{2} \gamma-1\right)+\left(1+\sigma_{2 t}^{2}\right)\right]^{2}}\left[1-\gamma \sigma_{2 t}^{2}\right] \\
+w_{1}^{(l)^{2}}\left|h_{1}^{(l)}\right|^{2}\left[1-\gamma \sigma_{1 t}^{2}\right] \\
+w_{1}^{(l)^{2}} \frac{2 \nu_{l}\left|h_{2}^{(l)}\right|^{2}\left|h_{1}^{(l)}\right|^{2}}{\nu_{l}\left|h_{2}^{(l)}\right|^{2}\left(\sigma_{2 t}^{2} \gamma-1\right)+\left(1+\sigma_{2 t}^{2}\right)}=\gamma \sigma_{i r}^{2}
\end{gathered}
$$

We note that $\Upsilon_{2}=\left[\nu_{l}\left|h_{2}^{(l)}\right|^{2}\left(\sigma_{2 t}^{2} \gamma-1\right)+\left(1+\sigma_{2 t}^{2}\right)\right]$ and $\Upsilon_{1}=\left[\nu_{l}\left|h_{1}^{(l)}\right|^{2}\left(\sigma_{1 t}^{2} \gamma-1\right)+\left(1+\sigma_{1 t}^{2}\right)\right]$ Finally,

$$
\left\{\begin{array}{l}
w_{1}^{(l)}=\sqrt{\frac{\gamma \sigma_{i r}^{2} \Upsilon_{2}^{2}}{\left|h_{1 j}^{(l)}\right|^{2}\left[\left(1+\sigma_{1 t}^{2}\right) \Upsilon_{2}^{2}+\left(\Upsilon_{2}-1+\sigma_{i r}^{2}\right)+2 \nu_{l} \Upsilon_{2}\left|h_{2 j}^{(l)}\right|^{2}\right]}} \\
w_{2}^{(l)}=\sqrt{\frac{\gamma \sigma_{i r}^{2} \Upsilon_{1}^{2}}{\left|h_{2}^{(l)}\right|^{2}\left[\left(1+\sigma_{2 t}^{2}\right) \Upsilon_{1}^{2}+\left(\Upsilon_{1}-1+\sigma_{i r}^{2}\right)+2 \nu_{l} \Upsilon_{1}\left|h_{1}^{(l)}\right|^{2}\right]}}
\end{array}\right.
$$

\section{Appendix C}

Taking into account the KKT conditions, we find that $\nu_{l}>0$ and $\lambda_{k}=0$ where $k \in 1,2, \ldots, M$. Thus,

$$
w_{k}^{(l)}=\frac{\nu_{l}\left|h_{k}^{(l)}\right|\left(\sum_{i=1}^{M} w_{i}^{(l)}\left|h_{i}^{(l)}\right|\right)}{\left(1+\sigma_{k t}^{2}\right)+\nu_{l} \gamma\left|h_{k}^{(l)}\right|^{2} \sigma_{k t}^{2}}
$$

Where

$$
\sum_{i=1}^{M} w_{i}\left|h_{i}\right|=\sum_{i=1}^{k-1} w_{i}\left|h_{i}\right|+\sum_{i=k+1}^{M} w_{i}\left|h_{i}\right|+w_{k}\left|h_{k}\right|
$$

To find the value of $\sum_{i=1}^{M} w_{i}^{(l)}\left|h_{i}^{(l)}\right|$ we replace (34) in (19), and it becomes:

$$
\begin{aligned}
& {\left[\sum_{i=1}^{M} w_{i}^{(l)}\left|h_{i}^{(l)}\right|\right]^{2}=\gamma\left[\sum_{i=1}^{M} \frac{\nu_{l}^{2}\left|h_{i}^{(l)}\right|^{4}\left(\sum_{i=1}^{M} w_{i}^{(l)}\left|h_{i}^{(l)}\right|\right)^{2}}{\left(1+\sigma_{i t}^{2}\right)+\nu_{l} \gamma\left|h_{i}^{(l)}\right|^{2} \sigma_{i t}^{2}} \sigma_{i t}^{2}+\sigma_{r}^{2}\right] } \\
= & \gamma \nu_{l}^{2}\left(\sum_{i=1}^{M} w_{i}^{(l)}\left|h_{i}^{(l)}\right|\right)^{2}\left[\sum_{i=1}^{M} \frac{\left|h_{i}^{(l)}\right|^{4}}{\left(1+\sigma_{i t}^{2}\right)+\nu_{l} \gamma\left|h_{i}^{(l)}\right|^{2} \sigma_{i t}^{2}} \sigma_{i t}^{2}\right]+\gamma \sigma_{r}^{2}
\end{aligned}
$$


Then,

$$
\left[\sum_{i=1}^{M} w_{i}^{(l)}\left|h_{i}^{(l)}\right|\right]^{2}=\frac{\gamma \sigma_{r}^{2}}{1-\gamma \nu_{l}^{2}\left[\sum_{i=1}^{M} \frac{\left|h_{i}^{(l)}\right|^{4}}{\left[\left(1+\sigma_{i t}^{2}\right)+\nu_{l} \gamma\left|h_{i}^{(l)}\right|^{2} \sigma_{i t}^{2}\right]^{2}} \sigma_{i t}^{2}\right]}
$$

Finally, equation (34) becomes:

$$
\begin{aligned}
w_{k}^{(l)} & =\frac{\nu_{l}\left|h_{k}^{(l)}\right|}{\left[\left(1+\sigma_{k t}^{2}\right)+\nu_{l} \gamma\left|h_{k}^{(l)}\right|^{2} \sigma_{k t}^{2}\right]} \\
& * \frac{\sigma_{r} \sqrt{\gamma}}{\sqrt{1-\gamma \nu_{l}^{2}\left[\sum_{i=1}^{M} \frac{\left|h_{i}^{(l)}\right|^{4}}{\left[\left(1+\sigma_{i t}^{2}\right)+\nu_{l} \gamma\left|h_{i}^{(l)}\right|^{2} \sigma_{i t}^{2}\right]^{2}} \sigma_{i t}^{2}\right]}}
\end{aligned}
$$

Now, the challenge is to find the value of $\nu_{l}$. Therefore, we multiply equation (34) by $\left|h_{k}\right|$, and obtain:

$$
\left|h_{k}^{(l)}\right| w_{k}^{(l)}=\frac{\nu_{l}\left|h_{k}^{(l)}\right|^{2}\left(\sum_{i=1}^{M} w_{i}^{(l)}\left|h_{i}^{(l)}\right|\right)}{\left(1+\sigma_{k t}^{2}\right)+\nu_{l} \gamma\left|h_{k}^{(l)}\right|^{2} \sigma_{k t}^{2}}
$$

After that, we compute the sum of all the resulting equations, we obtain:

$$
\begin{aligned}
& \sum_{k=1}^{M}\left|h_{k}^{(l)}\right| w_{k}^{(l)}=\sum_{k=1}^{M} \frac{\nu_{l}\left|h_{k}^{(l)}\right|^{2}\left(\sum_{i=1}^{M} w_{i}^{(l)}\left|h_{i}^{(l)}\right|\right)}{\left(1+\sigma_{k t}^{2}\right)+\nu_{l} \gamma\left|h_{k}^{(l)}\right|^{2} \sigma_{k t}^{2}} \\
= & \left(\sum_{i=1}^{M} w_{i}^{(l)}\left|h_{i}^{(l)}\right|\right) \sum_{k=1}^{M} \frac{\nu_{l}\left|h_{k}^{(l)}\right|^{2}}{\left(1+\sigma_{k t}^{2}\right)+\nu_{l} \gamma\left|h_{k}^{(l)}\right|^{2} \sigma_{k t}^{2}}
\end{aligned}
$$

Then,

$$
\sum_{i=1}^{M}\left|h_{k}^{(l)}\right| w_{k}^{(l)}\left[1-\sum_{k=1}^{M} \frac{\nu_{l}\left|h_{k}^{(l)}\right|^{2}}{\left(1+\sigma_{k t}^{2}\right)+\nu_{l} \gamma\left|h_{k}^{(l)}\right|^{2} \sigma_{k t}^{2}}\right]=0
$$

Since $\sum_{k=1}^{M} h_{k}^{(l)} w_{k}^{(l)} \neq 0$, we obtain:

$$
\sum_{k=1}^{M} \frac{\left|h_{k}^{(l)}\right|^{2}}{1+\sigma_{k t}^{2}\left(1+\nu_{l} \gamma\left|h_{k}^{(l)}\right|^{2}\right)}=\frac{1}{\nu_{l}}
$$

This equation is not written in a closed-form solution. Therefore, it can be solved numerically using the function "fminsearch" (J.C.Lagarias et al. 1998).

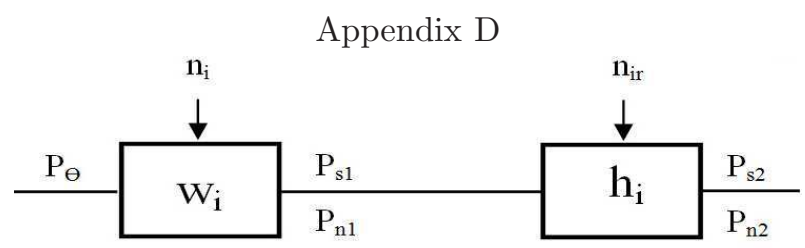

Figure 7 System Model

Assuming that we use real channels, the SNR corresponding to the $i^{\text {th }}$ sensor using the MMSE detector is given by (Figure 7):

$$
S N R_{i}=\frac{p_{s 2}}{p_{n 2}}=\frac{p_{s 1}\left|h_{i}\right|^{2}}{\sigma_{i r}^{2}+p_{n 1}\left|h_{i}\right|^{2}}
$$

Then,

$$
S N R_{i}=\frac{w_{i}^{2}\left|h_{i}\right|^{2} p_{\theta}}{\sigma_{i r}^{2}+\sigma_{i t}^{2}\left|h_{i}\right|^{2} w_{i}^{2}}
$$

Since $p_{\theta}=1$, then

$$
S N R_{i}=\frac{w_{i}^{2}\left|h_{i}\right|^{2}}{\sigma_{i t}^{2} w_{i}^{2}\left|h_{i}\right|^{2}+\sigma_{i r}^{2}}
$$

We express the $S N R_{i}$ in terms of transmission power, we obtain:

$$
\begin{gathered}
S N R_{i}=\frac{p_{i}\left|h_{i}\right|^{2}}{\sigma_{i t}^{2} p_{i}\left|h_{i}\right|^{2}+\left(1+\sigma_{i t}^{2}\right) \sigma_{i r}^{2}} \\
S N R_{i}=\frac{w_{i}^{2}\left|h_{i}\right|^{2}}{\sigma_{i t}^{2} w_{i}^{2}\left|h_{i}\right|^{2}+\sigma_{i r}^{2}}
\end{gathered}
$$

\section{References}

Abdellaoui, S. E., Fakhri, Y. \& Aboutajdine, D. (2009), 'Power and throughput optimization in ad-hoc cooperative mimo systems', International Conference in Multimedia Computing and Systems (8), 273-277.

Belmega, E. V., Lasaulce, S. \& Debbah, M. (2010), 'A survey on energy-efficient communications', Intl. Symp. on Personal, Indoor and Mobile Radio Communications, invited paper p. 289.

Chandrakasan, A. (1999), 'Design considerations for distributed micro-sensor systems', Custom Integrated Circuits Conference (CICC) p. 279286.

Chouhan, S., Bose, R. \& Balakrishnan, M. (2009), 'A framework for energy-consumption-based design space exploration for wireless sensor nodes', Computer-Aided Design of Integrated Circuits and Systems 28(7), 10171024.

Collin, L., Berder, O., Rostaing, P. \& Burel, G. (2004), 'Optimal minimum distance based precoder for mimo spatial multiplexing systems', IEEE Transactions on Signal Processing 52, 617-627.

Cui, S., Goldsmith, A. \& Bahai, A. (1999), 'Modulation optimization under energy constraints', IEEE International Conference on Communications 4, 28052811.

Cui, S., Goldsmith, A. J. \& Bahai, A. (2004), 'Energyefficiency of mimo and cooperative mimo techniques in sensor networks', IEEE Jour. On Selected Areas in Communications 22(6), 1089-1098.

Gesbert, D., Shafi, M., Shiu, D., P.Smith \& Naguib, A. (2003), 'From theory to practice: an overview of mimo space-time coded wireless systems', IEEE Journal on Selected Areas in Communications 47(21), 281-302.

Goudarzi, H. \& Pakravan, M. R. (2008), 'Equal power allocation scheme for cooperative diversity', 4th IEEE/IFIP International Conference 21, 1-5.

Hunter, T. E. \& Nosratinia, A. (2002), 'Cooperative diversity through coding', Proc. IEEE ISIT p. 220.

Hunter, T. E. \& Nosratinia, A. (2003), 'Performance analysis of coded cooperation diversity', Proc. IEEE ICC 4, 2688-2692. 
H.Wei, H. S. \& j. Kubokawa (1998), 'A decoupled solution of hydro-thermal optimal power flow problem by means of interior point method and network programming', IEEE Transactions on Power Systems 13, 286-293.

Jayaweera, S. K. (2004), 'Energy analysis of mimo techniques in wireless sensor networks', 38th Annual Conference on Information Sciences and Systems.

Jayaweera, S. K. (2006), 'Virtual mimo-based cooperative communication for energy-constrained wireless sensor networks', IEEE Trans. Wireless Communication 5, 984-989.

J.C.Lagarias, A.Reeds, J., Wright, M. H. \& Wright, P. E. (1998), 'Convergence properties of the nelder-mead simplex method in low dimensions', SIAM Journal of Optimization 9, 112-147.

Madhow, U. \& Honig, M. (1999), 'On the average nearfar resistance for mmse detection of direct sequence cdma signals with random spreading', IEEE Trans. Information Theory 45, 2039-2045.

Marzetta, T. L. \& Hochwald, B. M. (1999), 'Capacity of a mobile multiple-antenna communication link in rayleigh flat fading', IEEE Transactions on Information Theory 4, 139-157.

Namin, F. \& Nosratinia, A. (2008), 'Pragmatic lifetime maximization of cooperative sensor networks via a decomposition approach', Acoustics, Speech and Signal Processing (4), 30-17.

Nguyen, T. D., Berde, O. \& O.Sentieys (2007), 'Cooperative mimo schemes optimal selection for wireless sensor networks', IEEE 65th Vehicular Technology Conference (7), 85-89.

Nguyen, T. D., Berde, O. \& O.Sentieys (2008), 'Impact of transmission synchronization error and cooperative reception techniques on the performance of cooperative mimo systems', IEEE International Conference in Communications.

Nosratinia, A. \& Hedayat, A. (2004), 'Cooperative communication in wireless networks', IEEE Communications Magazine 42(10), 74-80.

S. Cui, A. J. G. \& Bahai, A. (2004), 'Energyefficiency of mimo and cooperative mimo techniques in sensor networks', IEEE Jour. On Selected Areas in Communications 22, 1089-1098.

S. Cui, J. J. Xiao, A. G. Z. Q. L. \& Poor, H. V. (2005), 'Energy-efficient joint estimation in sensor networks: analog vs. digital', Proc. ICASSP, Philadelphia p. 745748 .

Saxena, S., Bhatnagar, M. R. \& Kanaujia, B. K. (2010), 'Golden stbc-ofdm for mimo communications', International Conference on Computational Intelligence and Communication Networks pp. 519-522.

Sendonaris, A., Erkip, E. \& Aazhang, B. (2003), 'User cooperation diversity-part i: system description', IEEE Trans. Comm 51, 1927-1938.

Shafi, M., Gesbert, D., shan Shiu, D., Smith, P. \& Tranter, W. (2003), 'Guest editorial: Mimo systems and applications', IEEE Journal on Selected Areas in Communications 21, 277-280.
Shih, E. (2001), 'Physical layer driven protocol and algorithm design for energy-efficient wireless sensor networks', Proc. of the Seventh Annual ACM/IEEE International Conference on Mobile Computing and Networking p. 272286.

Winters, J. (1998), 'The diversity gain of transmit diversity in wireless systems with rayleigh fading', IEEE Transactions on Vehicular Technology 47(1), 119-123.

Y. Thomas Hou, Y. Shi, D. H. S. \& Wieselthier, E. J. (2007), 'Multicast communications in ad hocvnetworks using directional antennas: A lifetime-centric approach', IEEE Transactions on Vehicular Technology. 